\title{
Drosophila RecQ5 is required for efficient SSA repair and suppression of $\mathrm{LOH}$ in vivo
}

\author{
Yixu Chen ${ }^{1,2^{*}}$, Wen Dui ${ }^{1,2,3^{*}}$, Zhongsheng Yu ${ }^{1,2}$, Changqing $\mathrm{Li}^{1}$, Jun $\mathrm{Ma}^{1,3}$, Renjie Jiao ${ }^{1 凶}$ \\ ${ }^{1}$ State Key Laboratory of Brain and Cognitive Science, Institute of Biophysics, Chinese Academy of Sciences, 15 Datun Road, \\ Beijing 100101, China \\ ${ }^{2}$ Graduate School of the Chinese Academy of Sciences, Beijing 100080, China \\ ${ }^{3}$ Divisions of Biomedical Informatics and Developmental Biology, Cincinnati Children's Hospital Research Foundation, 3333 \\ Burnet Avenue, Cincinnati, OH 45229, USA \\ $\triangle$ Correspondence: rjiao@sun5.ibp.ac.cn
}

Received April 6, 2010 Accepted April 25, 2010

\section{ABSTRACT}

RecQ5 in mammalian cells has been suggested to suppress inappropriate homologous recombination. However, the specific pathway(s) in which it is involved and the underlining mechanism(s) remain poorly understood. We took advantage of genetic tools in Drosophila to investigate how Drosophila $\operatorname{Rec} Q 5$ (dRecQ5) functions in vivo in homologous recombination-mediated double strand break (DSB) repair. We generated null alleles of dRecQ5 using the targeted recombination technique. The mutant animals are homozygous viable, but with growth retardation during development. The mutants are sensitive to both exogenous DSB-inducing treatment, such as gamma-irradiation, and endogenously induced double strand breaks (DSBs) by I-Sce I endonuclease. In the absence of $d R e c Q 5$, single strand annealing (SSA)-mediated DSB repair is compromised with compensatory increases in either inter-homologous gene conversion, or non-homologous end joining (NHEJ) when inter-chromosomal homologous sequence is unavailable. Loss of function of $d R e c Q 5$ also leads to genome instability in loss of heterozygosity (LOH) assays. Together, our data demonstrate that $d R e c Q 5$ functions in SSA-mediated DSB repair to achieve its full efficiency and in suppression of LOH in Drosophila.

KEYWORDS Drosophila RecQ5, double strand break repair, homologous recombination, nonhomologous end joining, single strand annealing, $\operatorname{Rec} Q$ helicase

\section{INTRODUCTION}

DNA breaks need to be properly repaired to ensure the integrity of an organism's genome. Un-repaired DNA breaks are deleterious to cells during cell divisions leading to cell cycle arrest and eventually lethality of organisms (JohnsonSchlitz and Engels, 2006; Preston et al., 2006). Our understanding of double strand DNA break (DSB) repair in eukaryotes has been greatly facilitated by the use of sitespecific endonucleases, such as I-Sce I, a rare yeast cutter that is also active when expressed in other eukaryotic cells such as mammalian and Drosophila cells (Plessis et al., 1992; Rouet et al., 1994; Johnson-Schlitz et al., 2007; Wei and Rong, 2007). There are three major DSB repair pathways in Drosophila both in mitotic and meiotic cells (Adams et al., 2003; Rong and Golic, 2003; Preston et al., 2006; Wei and Rong, 2007). Single strand annealing (SSA), which has been shown to be the first choice for DSB repair in Drosophila (Preston et al., 2006; Wei and Rong, 2007), uses homologous sequences that flank the break. SSA repair always results in a deletion that uncovers the DNA between the two stretches of homologous sequence (Preston et al., 2006). Non-homologous end joining (NHEJ) is usually error prone and often creates small deletions or insertions at the break site, although it can also re-ligate the broken ends perfectly without causing any mutations. Recombinational repair between homologous chromosomes through either synthesis dependent strand annealing (SDSA) or double holiday junction (DHJ) results in gene conversion (GC), while crossovers produce loss of heterozygosity (Rong and Golic, 2003; Preston et al., 2006). These different pathways

\footnotetext{
*These authors contributed equally to this work
} 
represent choices for a cell to repair a given DSB with preferences that are dependent on the genomic context of where the DSB occurs, the developmental stages and likely additional factors such as the proximity to an essential gene (Preston et al., 2006; Wei and Rong, 2007). RAD52 and related proteins, such as Rad50, Rad51, Rad54, Rad59 and Mre11, are known to be involved in regulating the gene conversion process (Ghabrial et al., 1998; Wei and Rong, 2007). Furthermore, several conserved proteins have been demonstrated to regulate NHEJ, including the Ku70, Ku80 and the DNA ligase IV (Kusano et al., 2001; Wei and Rong, 2007). However, the factors that participate in SSA in Drosophila remain to be elucidated.

RecQ helicase family is a group of ATP-dependent DNA helicases that maintains genome stability by regulating DNA recombination, repair and replication (Bachrati and Hickson, 2008; Chu and Hickson, 2009). The Drosophila genome encodes three RecQ helicases, dBLM, dRecQ4 and dRecQ5 (Kusano et al., 1999; Sekelsky et al., 1999; Kusano et al., 2001; Kawasaki et al., 2002; Adams et al., 2003; McVey et al., 2007; Trowbridge et al., 2007; Wu et al., 2008; Nakayama et al., 2009; Xu et al., 2009). In addition, DmWRNexo was recently reported to be homologous to the human WRN exonuclease domain (Saunders et al., 2008; Boubriak et al., 2009). Loss of $d B L M$ causes sterility in flies (Kusano et al., 1999; Kusano et al., 2001). dBLM mutants also exhibit other defects including impaired DNA synthesis during homologous repair (HR) (Adams et al., 2003), reduced frequency in homologous repair from the homologous chromosome (HRh) with a concurrent increase in SSA frequency (JohnsonSchlitz and Engels, 2006; Kappeler et al., 2008), and an elevated crossovers due to defects in the dissolution of holiday junctions during DSB repair (Johnson-Schlitz and Engels, 2006). We and others have shown recently that dRecQ4 is essential for Drosophila development and involved in DNA replication and repair (Wu et al., 2008; Xu et al., 2009). In contrast, despite the well characterized biochemical properties of dRecQ5, including strand annealing activities and the unwinding of the substrates of 3' flaps, three-way junctions, forks, and three-strand junctions, some of which are unique to RecQ5 among the RecQ members (Sekelsky et al., 1999; Jeong et al., 2000; Kawasaki et al., 2002; Nakayama et al., 2009), the in vivo functions of $d R e c Q 5$ remain largely unknown.

RecQ5 is a conserved gene in all multicellular organisms ranging from worms to mammals. In C. elegans, RNAi against RecQ5 increases the animal's sensitivity to ionizing irradiation (Jeong et al., 2003). In mouse ES cells, RecQ5 is shown to suppress both crossovers and the repair of I-Sce I-induced double-strand breaks by homologous recombination (Hu et al., 2005). Loss of Drosophila RecQ5 leads to chromosomal aberrations during early embryonic development (Nakayama et al., 2009). RecQ5 in mammalian cells has been shown to suppress inappropriate homologous recombination by disrupting
RAD51 recombinase-mediated presynaptic filaments (Hu et al., 2007). It localizes to the sites of double strand breaks by interacting with MRN complex (Zheng et al., 2009). To understand how RecQ5 precisely functions in the DSB repair pathways, we made use of the Drosophila DSB systems that were developed in Engels and Rong labs (Preston et al., 2006; Wei and Rong, 2007), which can detect simultaneously the usage of different DSB repair pathways. We examined and compared the changes of induced DSB repair in the presence or absence of $d R e c Q 5$. We show that $d R e c Q 5$ is specifically required for full efficiency of SSA-mediated DSB repair and $d R e c Q 5$ mutation leads to genome instability as assayed by loss of heterozygosity experiments.

\section{RESULTS}

\section{Generation of $d R e c Q 5$ mutants and molecular characterization}

To gain a better understanding of the precise molecular functions of $d R e c Q 5$ in Drosophila, we set off to knock out the $d R e c Q 5$ gene in flies by the specific ends-in gene targeting technique (Fig. 1A). A donor construct that contains an FRTflanked $\sim 8 \mathrm{~kb} d \operatorname{Rec} Q 5$ genomic fragment, which was modified with intended mutations at the ATG site and an artificial I-Sce I cutting site $\sim 2 \mathrm{~kb}$ downstream of the ATG, was inserted into the fly genome through $P$ element mediated transformation (see materials and methods for details). After two rounds of homologous recombination, the endogenous wild type copy of $d R e c Q 5$ locus was replaced by the designed mutant copy of $d R e c Q 5$ (Fig. 1A and 1B). Three alleles were obtained after the reduction step of gene targeting, which were confirmed to contain the designed mutations as judged by the introduction of Not I restriction site (Fig. 1C). All three alleles exhibit identical phenotypes of being homozygous viable and fertile. One of these alleles, designated as $d R e c Q 5^{5}$, was further confirmed by sequencing for the mutation (Fig. 1B) and demonstrating the absence of all three different isoforms of dRecQ5 protein (Fig. 1B and 1D). This allele was used in further studies described in the following sections.

\section{dRecQ5 is required for normal development and mitosis in Drosophila}

Although $d R e c Q 5$ null mutant flies are homologous viable and fertile without obvious morphological defects, careful examination of the mutant animals revealed that over $80 \%$ of the mutants were smaller than the wild type at same developing time points of the larval stages particularly at 72 and $96 \mathrm{~h}$ after egg deposition (AED). Images of typical mutant and wild type larvae are shown in pairs for the same developing time points in Fig. $2 \mathrm{~A}$. Fig. $2 \mathrm{~B}$ and $2 \mathrm{C}$ show that the mutants develop more slowly than the wild type. At $114 \mathrm{~h}$ 

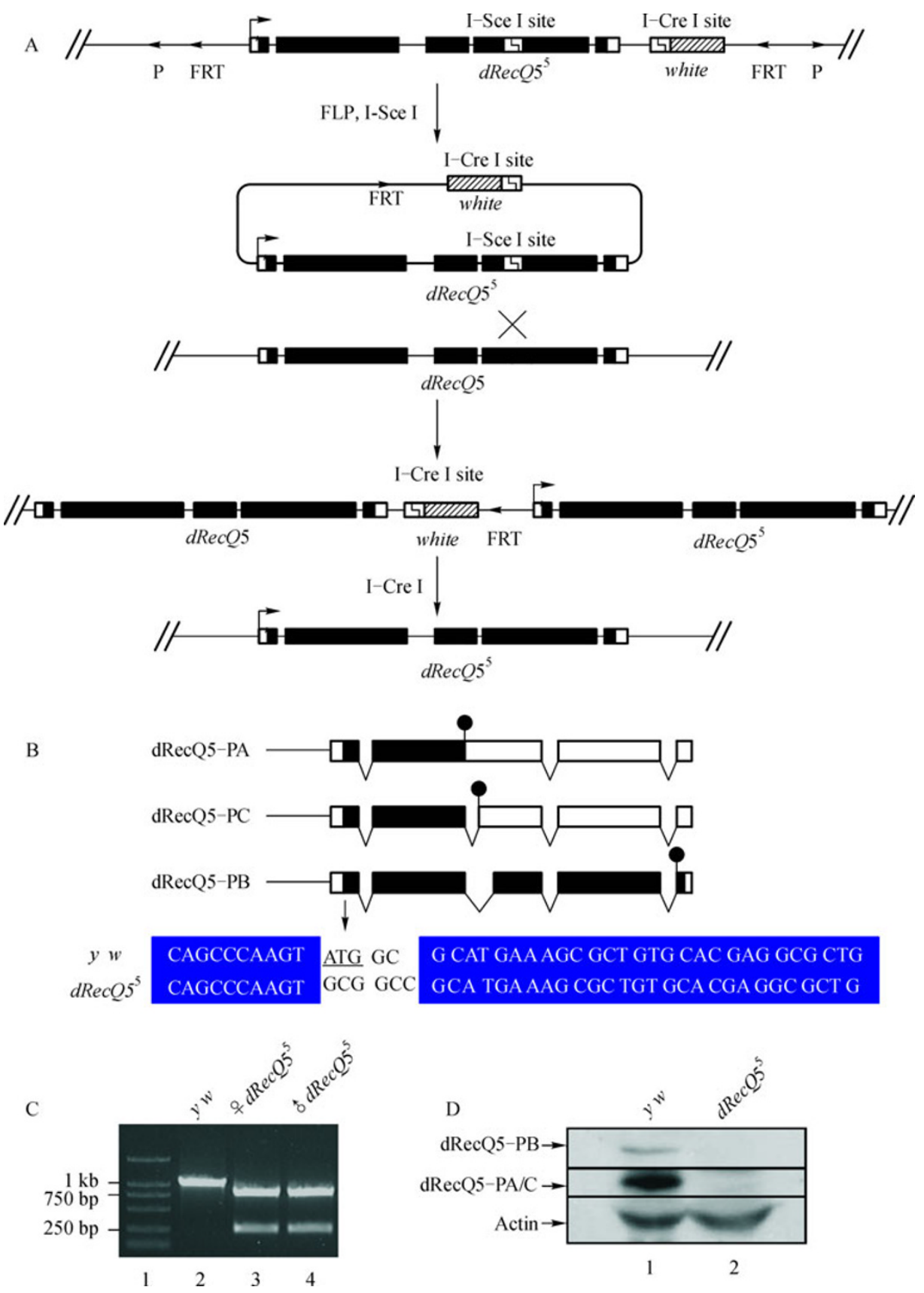

Figure 1. Generation of $d \operatorname{Rec} Q 5$ mutant $\left(d \operatorname{Rec} Q 5^{5}\right)$ : strategy and molecular characterization. (A) Schematic diagram of $d R e c Q 5$ genomic locus and gene targeting strategy. A mutant $d R e c Q 5$ (* indicates the mutation site at the start codon) and the marker gene $w^{+}$are circularized from the transgenic genome by FLP recombinase and linearized by the yeast restriction endonuclease I-Sce I before ends-in recombination resulting in a duplication of the dRecQ5 locus (one wild type and the other mutant). Upon I-Cre I cutting and repairing via homologous recombination, the $d R e c Q 5$ mutant can be selected with the loss of white marker gene. (B) Sequence comparison of $d R e c Q 5^{5}$ and the wild type (WT) indicating that the expected ATG (underlined) mutation to GCG (yielding a Not I cutting site) and a frame shift of the coding sequence in $d R e c Q 5^{5}$. Three mRNA splice isoforms of $d R e c Q 5$ (dRecQ5-PA, -PB and-PC) are shown above the sequence comparison. (C) PCR in combination with Not I digestion (see materials and methods for details) shows that the artificial Not I site in the $d R e c Q 5^{5}$ gives two bands of the PCR products (lane 3 and 4 ) while the PCR products from wild type flies cannot be cut by Not I (lane 2). Lane 1 is DNA ladders. (D) Western blot showing that the $53 \mathrm{kDa}$ short forms of dRecQ5-PA and dRecQ5-PC, and the $121 \mathrm{KD}$ form of dRecQ5-PB become undetectable in $d R e c Q 5^{5}$ (lane 2). Lane 1 is the wild type control. The $43 \mathrm{kDa}$ actin band is loading control. Molecular weights are indicated on the left of the panel. 
A

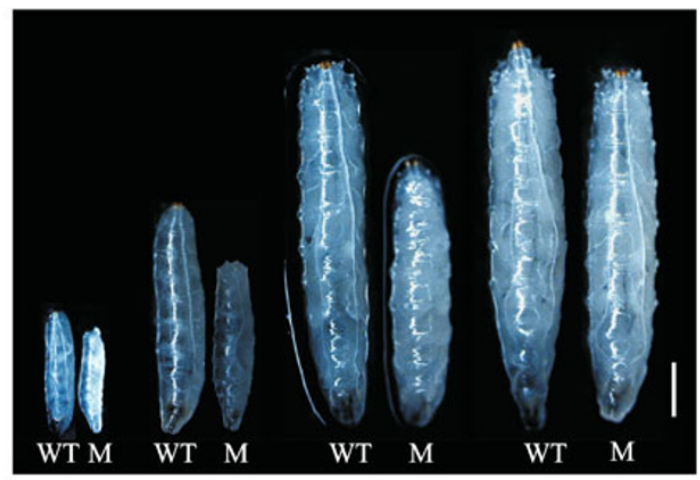

$48 \mathrm{~h} \quad 72 \mathrm{~h} \quad 96 \mathrm{~h} \quad 114 \mathrm{~h}$

B

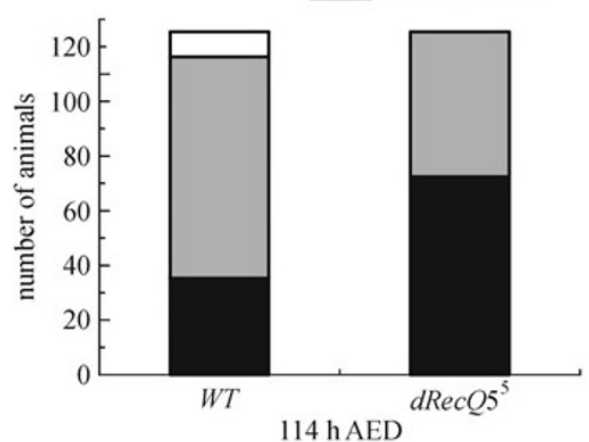

D

$W T$

$d \operatorname{Rec} Q 5^{5}$
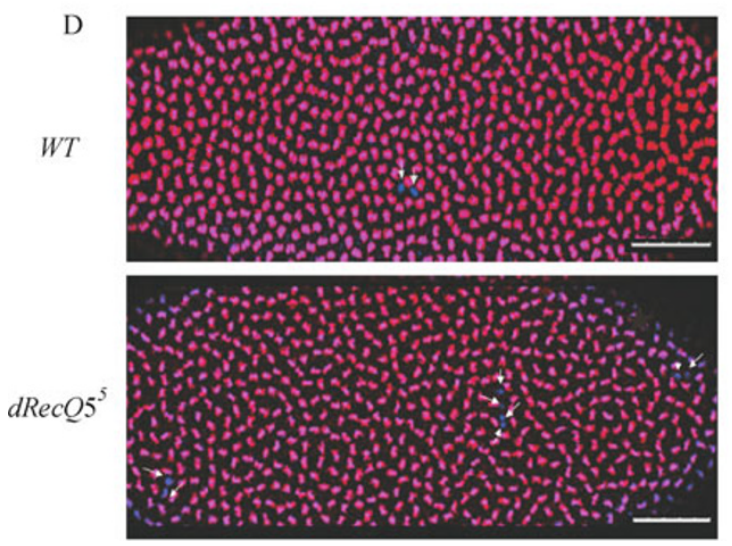

$\mathrm{C}$
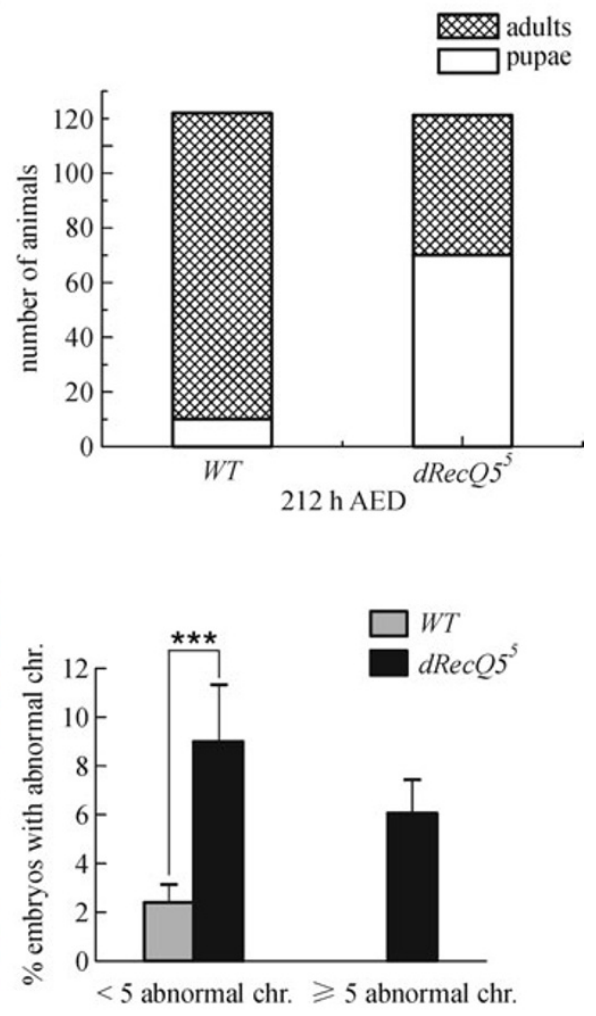

Figure 2. Cellular and growth phenotypes of $d R e c Q 5$ homozygous mutants. (A) Comparison of wild type and $d R e c Q 5^{5}$ animals at indicated time points of larval stages. WT indicates wild type larvae and M indicates mutant larvae. Scale bar, $600 \mu \mathrm{M}$. (B) Fractions of feeding larvae, wandering larvae and pupae at $114 \mathrm{~h}$ after egg deposition (AED) for both $d R e c Q 5^{5}$ and wild type. (C) Fractions of pupae and adults at $212 \mathrm{~h}$ AED for both $d R e c Q 5^{5}$ and wild type. (D) Loss-of-function of $d R e c Q 5$ leads to spontaneous mitotic defects with increased frequency of aberrant chromosomes in early embryos (embryos that contain less than 5 mitotic abnormal chromosomal clusters (abnormal chr.) increased from $\sim 2 \%$ to $\sim 9 \%$, while embryos that contain 5 and more mitotic abnormal chromosomal clusters increased from 0 to $\sim 6 \%)$. Wild type and mutant embryos $(0-2 \mathrm{~h})$ were collected, fixed and stained for DNA (Topro3: blue) and phosphorylated histone H3 (PH3: red). Abnormal wild-type (above) and dRecQ5 mutant (below) embryos at cycle 13 (prophase/metaphase) are shown on the left. Arrows indicate nuclei with mitotic defects. Scale bar, $50 \mu \mathrm{M}$. Statistic analysis is shown on the right. $p<0.01$, Student's t-test. More than 200 embryos were scored for each category. 
$A E D$, the number of the mutant animals that enter wandering larval stage was about half of that of wild type animals (Fig. 2B). Similarly, the number of adult mutants was also about half of that of the wild type at $212 \mathrm{~h}$ AED (Fig. 2C). The observed developmental delay of the $d R e c Q 5$ mutants implies that under the same culture conditions, the mutant animals may encounter more endogenous stress, such as unrepaired DNA damages, than the wild type animals during development. Fig. 2D shows that in early stages of the embryonic development mutant embryos exhibit increased frequency to have abnormal mitotic chromosomes and irregular nuclei as compared with the wild type embryos (see Figure legends for details). These defects are likely reflective of accumulation of endogenous DNA damages as recently reported by Nakayama and colleagues (Nakayama et al., 2009), suggesting that $d R e c Q 5$ mutant animals may be more vulnerable to endogenous DNA damage stress, and consequently exhibit developmental retardation.

\section{dRecQ5 mutants show different sensitivity to double strand breaks (DSBs) generated in different assays}

To determine directly whether $d R e c Q 5$ mutants are more sensitive to DNA damages, particularly to double strand breaks, we treated the third instar larvae of wild type and $d R e c Q 5^{5}$ with gamma irradiation at different doses. The treated animals were then allowed to recover and to further develop at normal culture conditions for different periods of time (see the materials and methods and figure legends for details). Our results show that the mutants exhibit a significantly lower survival rate than wild type controls (Fig. 3A), suggesting that $d \operatorname{Rec} Q 5$ mutants are more sensitive to
DSB-inducing treatment. To further demonstrate this point, we specifically induced endogenous DSBs with I-Sce I cut at the [w/w]4A transgene that contains an I-Sce I recognizing sequence (Preston et al., 2006; Wei and Rong, 2007). The expression of I-Sce I enzyme was induced under the control of a ubiquitous promoter (Preston et al., 2006). The ratio of [W/w]4A Sco/CyO[UIE] offspring flies (as the DSB-occurring fraction) to offspring $S p / C y O$ flies (as the fraction without DSB) was used as the survival rate (Fig. 3B). In wild type animals, this ratio is almost $100 \%$, indicating that the artificially induced DSBs can be efficiently repaired. However, in the absence of $d R e c Q 5$, the survival rate dropped to about $60 \%$, demonstrating a compromised DSB repair system in dRecQ5 mutant animals.

We employed another assay, termed yellow reconstitution (Takeuchi et al., 2007), to examine the sensitivity of $d R e c Q 5$ mutant animals to endogenously induced DSBs. As illustrated in Fig. 4A and 4B, a defective yellow gene, which consists of only the 5' and 3' segments of the wild type yellow gene separated by the I-Sce I cutting sequence, was used for generating DSB. Upon the cut of I-Sce I endonuclease, the broken ends would either find the endogenous yellow ${ }^{1}$ homologous sequence to initiate a gap repair, leading to a repaired yellow gene with darker $\left(y^{+}\right)$body color (Fig. 4C), or simply be rejoined by non-homologous end joining (NHEJ). The un-repaired broken ends would cause lethality of the flies. Fig. 4E shows that wild type and dRecQ5 mutants exhibit, surprisingly, similar survival rate (lethality) upon the induction of DSBs (F-R3, 60F5 of the second chromosome) by I-Sce I endonuclease. However, when the DSBs occur on the $X$ chromosome (F1-5, 7E7 of the $X$ chromosome), the survival rate exhibited significant difference in the presence or
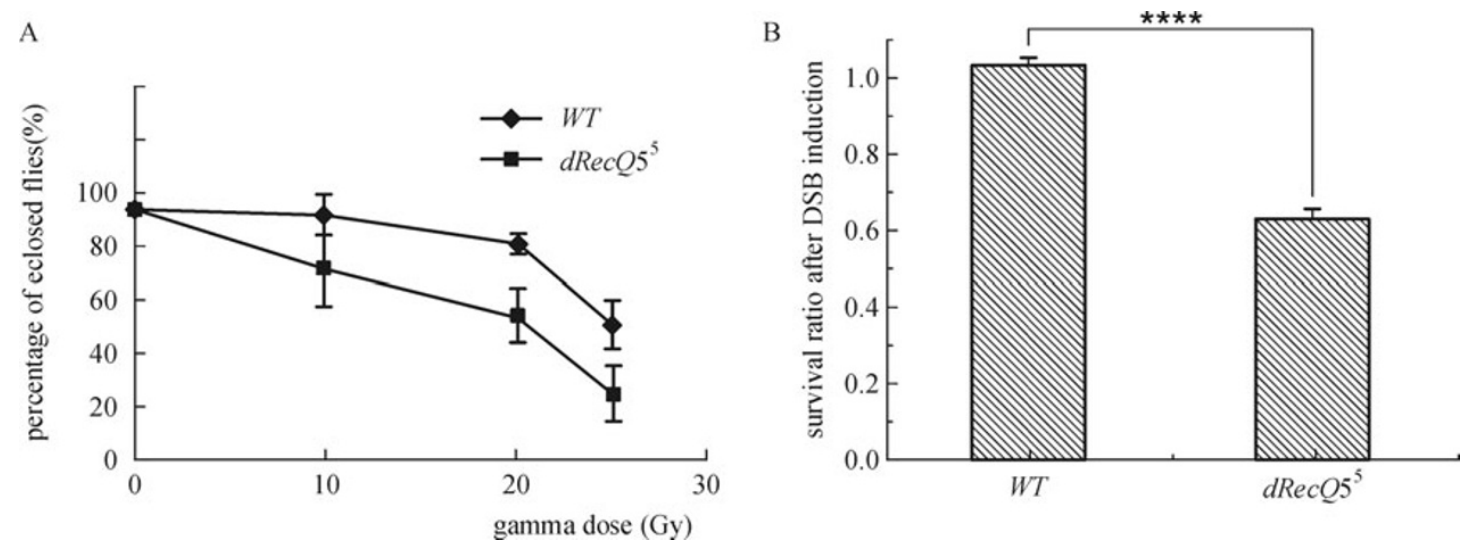

Figure 3. $d R e c Q 5$ mutants are sensitive to agents that cause DSBs. (A) The third instar larvae of wild type and $d R e c Q 5^{5}$ were treated with gamma irradiation at the indicated doses and allowed to recover and further develop at normal culture conditions. The mutants show significantly lower survival rate than the wild type control. (B) The survival rate is significantly reduced for the $d R e c Q 5^{5}$ than the wild type flies upon the endogenous DSBs induction by I-Sce I endonuclease cutting at the [w/w] $4 A$ transgene (Preston et al., 2006; Wei and Rong, 2007). [W/w]4A Sco/CyO females were mated to Sp/CyO[UIE] males. [W/w]4A Sco/CyO[UIE] flies in the offspring indicate DSB-occurring fraction while $S p / C y O$ flies were scored as the endogenous control. More than 5000 flies in total were scored. Data were analyzed by Student's $t$ test and presented as mean \pm SEM with * for $p<0.05$, ${ }^{* *}$ for $p<0.01$, and ${ }^{* * *}$ for $p<0.001$. 
absence of dRecQ5 (Fig. 4D, columns for total). It is worthwhile to note that, the survival rate difference in males is much more dramatic than in females. A clear difference in the ability to survive the induced DSBs also exists between males and females even in wild type background (Fig. 4D, columns for females and males). Together, the results of

A

FI-5 model

females
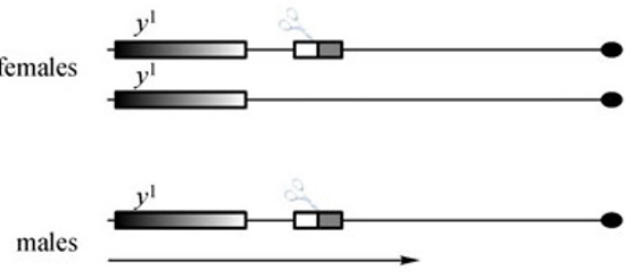

$\mathrm{B}$

F-R3 model
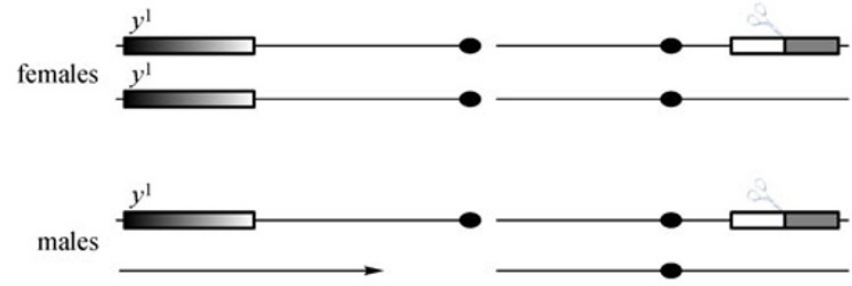

C
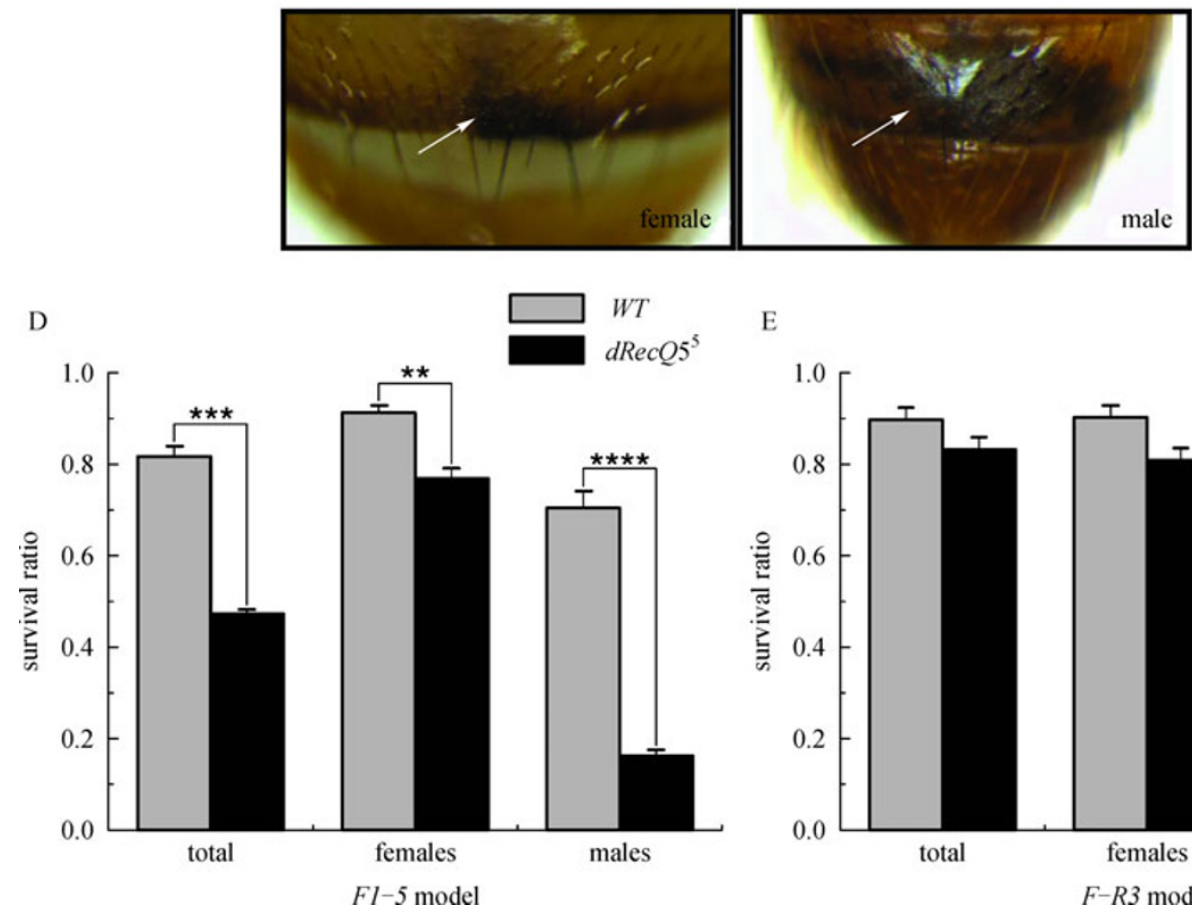

E $W T$

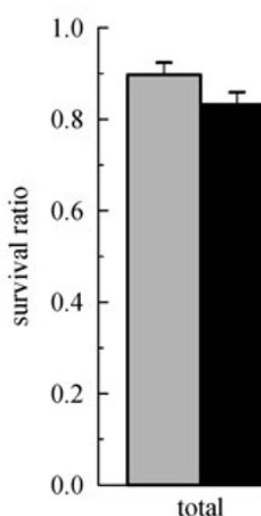

total

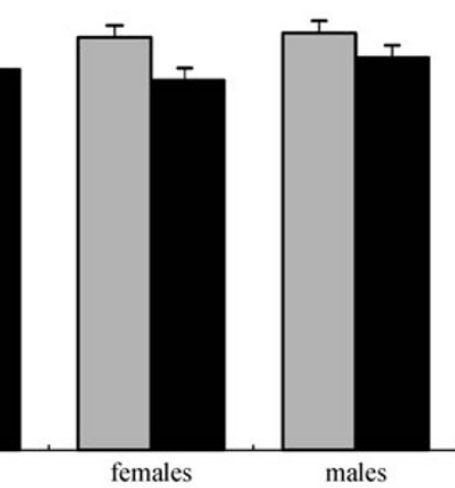

$F-R 3$ model

Figure 4. $d R e c Q 5$ mutants show different sensitivity to DSBs generated in different assays. (A and $B$ ) Schematic diagrams (not to the scale) of the $y^{+}$reconstitution assays $F 1-5$ and $F-R 3$ (Takeuchi et al., 2007) for gap repair. Both $F 1-5$ and $F-R 3$ trangenes contain an incomplete yellow gene containing only the 5' and 3' segments that are separated by an I-Sce I cutting site. F1-5 is located at 7E7 on the X chromosome while F-R3 is located at 60F5 on the second chromosome. (C) Reconstituted $y^{+}$somatic clones (arrows) in both females (left) and males (right). (D) Males are more sensitive to I-Sce I endonuclease than females in the absence of $d R e c Q 5$ (F1-5 assay). (E) Females and males show similar sensitivities to I-Sce I induced DSBs regardless of the presence of $d R e c Q 5$ in F-R3 assay. F-R3/CyO females were mated to Sp/CyO[UIE] males in the presence or absence of $d R e c Q 5$. $F-R 3 / C y O[U I E]$ flies represent the DSB-occurring fraction, and the Sp/CyO flies are used as an endogenous control. More than 5000 flies in total were scored. Data were analyzed by Student's $t$ test and presented as mean \pm SEM with * for $p<0.05$, ${ }^{* *}$ for $p<0.01$, and ${ }^{* * *}$ for $p<0.001$. 
endogenously induced DSB assays suggest that, depending on where the DSB occurs and what kind of template sequence is available for repair, the induced DSBs may cause different degrees of animal lethality (see discussion).

\section{SSA repair pathway is impaired in the absence of $d R \operatorname{Rec} Q 5$}

To further investigate the role of $d R e c Q 5$ in DSB repair pathway(s), we took advantage of the well-established w/w repair assay that can measure simultaneously the outcome of different DSB repair pathways (Wei and Rong, 2007). As illustrated in Fig. 5A, flies that harbor the $w / w$ reporter construct (a complete mini-white and a truncated 3' mini-white segment separated by a I-Sce I cutting sequence) and the ISce I endonuclease transgene may, in principle, have four different repair pathways for the I-Sce I induced DSBs, which can be detected by crossing again these flies with the ones that contain the I-Sce I endonuclease. Inter-sister chromatid GC and perfect non-homologous end joining (NHEJ without any errors) will reconstitute the intact $w / w$ construct that can be re-cut by I-Sce I endonuclease in the somatic cells, leading to mosaic eyes after different DSB repair in different cells (Fig. 5A). Imperfect NHEJ destroys the I-Sce I cutting site making the repaired $w / w$ construct uncuttable by I-Sce I in
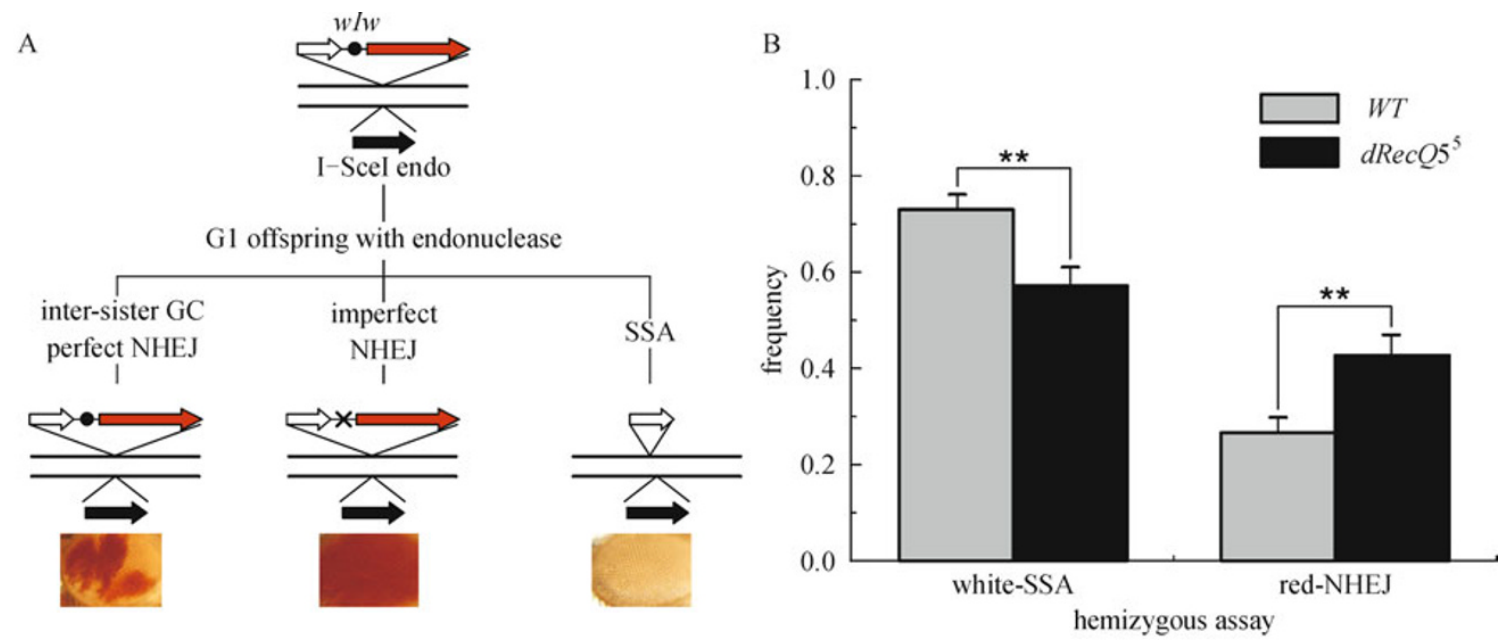

C

D
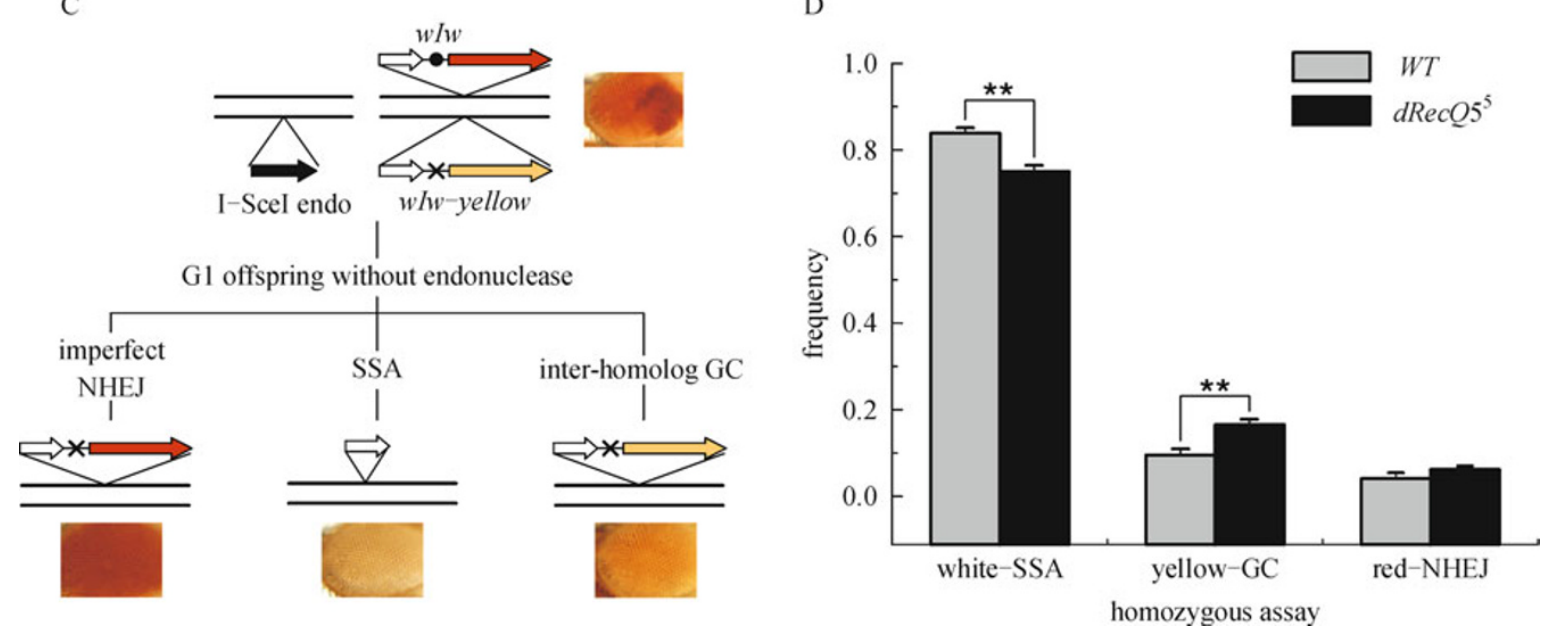

Figure 5. $d R e c Q 5$ mutation impairs SSA mediated DSB repair. (A) Schematic illustration of the hemizygous assay. SSA and NHEJ frequencies in WT and $d R e c Q 5$ mutant backgrounds are shown in B. (C) Schematic illustration of the homozygous assay. The w/w insertion contains a part of the 3' portion of $w^{+}$(to the left of the I-Sce I site) and a copy of the functional mini-white. G0 generation that carries w/W and UIE exhibits mosaic eyes. Among the G1 offspring, three kinds of eyes represent the results of the indicated repair pathways. $\times$ represents a mutated I-Sce I site due to imperfect NHEJ. (D) SSA, inter-homolog GC and NHEJ frequencies in WT and $d R e c Q 5$ mutant background are shown. More than 200 flies were scored for each genotype. Data were analyzed by Student's $t$ test and presented as mean \pm SEM with * for $p<0.05$, ** for $p<0.01$, and ${ }^{* * *}$ for $p<0.001$. 
somatic cells, thus producing red-eyed flies (Fig. 5A). The direct repeats of the 3 ' segments of the mini-white gene flanking the I-Sce I cutting sequence will lead to SSA repair upon the action of the I-Sce I endonuclease, which results in the loss of the intact white gene, producing white-eyed flies (Fig. 5A). Fig. 5B shows that the efficiency of SSA in dRecQ5 animals was reduced by about $16 \%$ when compared with wild type animals, suggesting a role of $d R e c Q 5$ in the SSA repair pathway. Along with the reduction of SSA repair, an increase in imperfect NHEJ was observed (Fig. 5B), suggesting that a compensatory repair is triggered in the $d R e c Q 5$ mutant background.

\section{GC is preferred over non-homologous end joining (NHEJ) for flies to repair DSBs in the absence of $d R e c Q 5$}

In order to examine how GC mediated DSB repair is affected by $d R e c Q 5$ mutation, a modified w/w system, termed as [w/w]yellow, was employed (Wei and Rong, 2007). As explained in Fig. $5 \mathrm{C}$, in addition to the $w / W$ and I-Sce I transgenes, another construct, [w/w]yellow, was implanted in the same flies, which can provide a gene conversion template for the broken w/w construct. However, there is no I-Sce I cutting sequence in [w/w]yellow, in which the mini-white gene contains a point mutation that makes the red pigmentation significantly weaker than the wild type mini-white gene, thus creating orange-eyed flies when present in the fly genome. The outcome of three major repair pathways after such flies are crossed to $y w$ flies is shown in Fig. 5C. In addition to imperfect NHEJ and SSA, which appear also in the w/w assay, GC through homologous recombination between homologous chromosomes will give yellow-eyed offspring (Fig. 5C). Fig. 5D shows that in the [w/w]yellow assay, similar to the result from w/w assay, SSA is reduced in the absence of $d R e c Q 5$ compared with wild type. Unlike in the w/w assay, the imperfect NHEJ repair frequency remains unchanged regardless of the presence or absence of $d R e c Q 5$ function. However, the gene conversion between the homologous chromosome-mediated repair increased significantly in the absence of $d R e c Q 5$ (Fig. 5D), suggesting that these flies prefer to use GC than NHEJ to repair DSBs when there is homologous template available.

\section{dRecQ5 mutation leads to increased loss of heterozygosity (LOH)}

To determine whether $d R e c Q 5$ participates in the formation of crossover between homologous chromosomes, which is one of the driving forces for loss of heterozygosity and genome instability (Saunders et al., 2008) (Fig. 6A and 6D), we took advantage of two assays for $\mathrm{LOH}$ detection. The first assay involves $m h w^{1}$ mutation, homozygotes of which show extra hairs on the wing (Fig. 6B). Somatic clones of $m h w^{1}$ may occur in the heterozygous animals if spontaneous homologous recombination happens between the two homologous chromosomes, leading to the loss of heterozygosity due to crossovers (Fig. 6A). In the absence of $d R e c Q 5$, the frequency of $m h w^{1}$ clone occurrence increased significantly compared with wild type (Fig. 6C). In the second assay of $\mathrm{LOH}$ detection, $w^{+}$was used as a marker to monitor the loss of the wild type allele in the presence or absence of $d R e c Q 5$ (Fig. 6D). Fig. 6D shows that in $d R e c Q 5$ mutant background, in all the testing tubes (7 out of 7 ) mosaic eyed flies were observed while in wild type flies, no mosaic eyed flies (LOH) were found ( 0 out of 7 ). These results suggest that $d R e c Q 5$ may be important for suppressing crossovers during homologous recombination, thus maintain the heterozygosity and genome stability.

\section{DISCUSSION}

In this study we show that Drosophila RecQ5 has a role in SSA repair upon the stress of induced endogenous DSBs by I-Sce I endonuclease. However, unlike the other Drosophila RecQ members, $d R e c Q 5$ is neither essential for the animal development and cell survival as $d R e c Q 4$ is ((Wu et al., 2008; Xu et al., 2009) and this study), nor required for animal fertility as $d B L M$ is ((Kusano et al., 2001; Adams et al., 2003; Johnson-Schlitz and Engels, 2006) and this study). Therefore, the $d R e c Q 5$ gene is a functionally divergent RecQ family member in Drosophila compared with other RecQ members.

SSA has been shown to be the most frequently used pathway for DSB repair in Drosophila (Preston et al., 2006; Wei and Rong, 2007). It is thought that one of the functions of SSA in vivo is to reduce any unintentionally duplicated DNA sequence to the original copy in the genome. In Drosophila a natural substrate for SSA may come from the hybrid element insertion (HEI) process: a P-element located on one sister chromatid can recombine with a nearby locus on the homologous chromosome, resulting in a deletion and a duplication product. The generated duplication becomes prone to undergoing SSA to preserve the original genome sequence so as to maintain genomic stability. Until now, the factors that are involved in this process have remained unclear in Drosophila. Those that are involved in SSA repair in mammalian cells, such as Rad59, Rad52, MSH2 and $\mathrm{MSH} 3$, are mostly absent in the Drosophila genome (Wei and Rong, 2007; Kappeler et al., 2008); only the MSH2 ortholog, spel1, exists in Drosophila. In our current study we show that $d R e c Q 5$ mutation affects SSA repair, suggesting that $d R e c Q 5$ plays at least a modulator role in the process of repairing artificially induced DSBs. In the absence of $d R e c Q 5$ the SSA repair frequency decreases in both $w / w$ and [w/w]yellow assays. The w/w assay offers no homologous template for gene conversion, in which NHEJ increases but SSA decreases in $d R e c Q 5$ mutants (Fig. 5B). However, once there is homologous sequence available for gene conversion repair, as shown in the [w/w]yellow assay, GC increases while 
NHEJ remains unaffected in $d R e c Q 5$ mutants (Fig. 5D). These results demonstrate that: (1) error-free (GC) pathway is a preferred mechanism to repair DSBs in flies over the error- prone (NHEJ) pathway when dRecQ5 function is absent; (2) Gene conversion and non-homologous end joining are not impaired by $d R e c Q 5$ loss-of-function. The mechanism of how
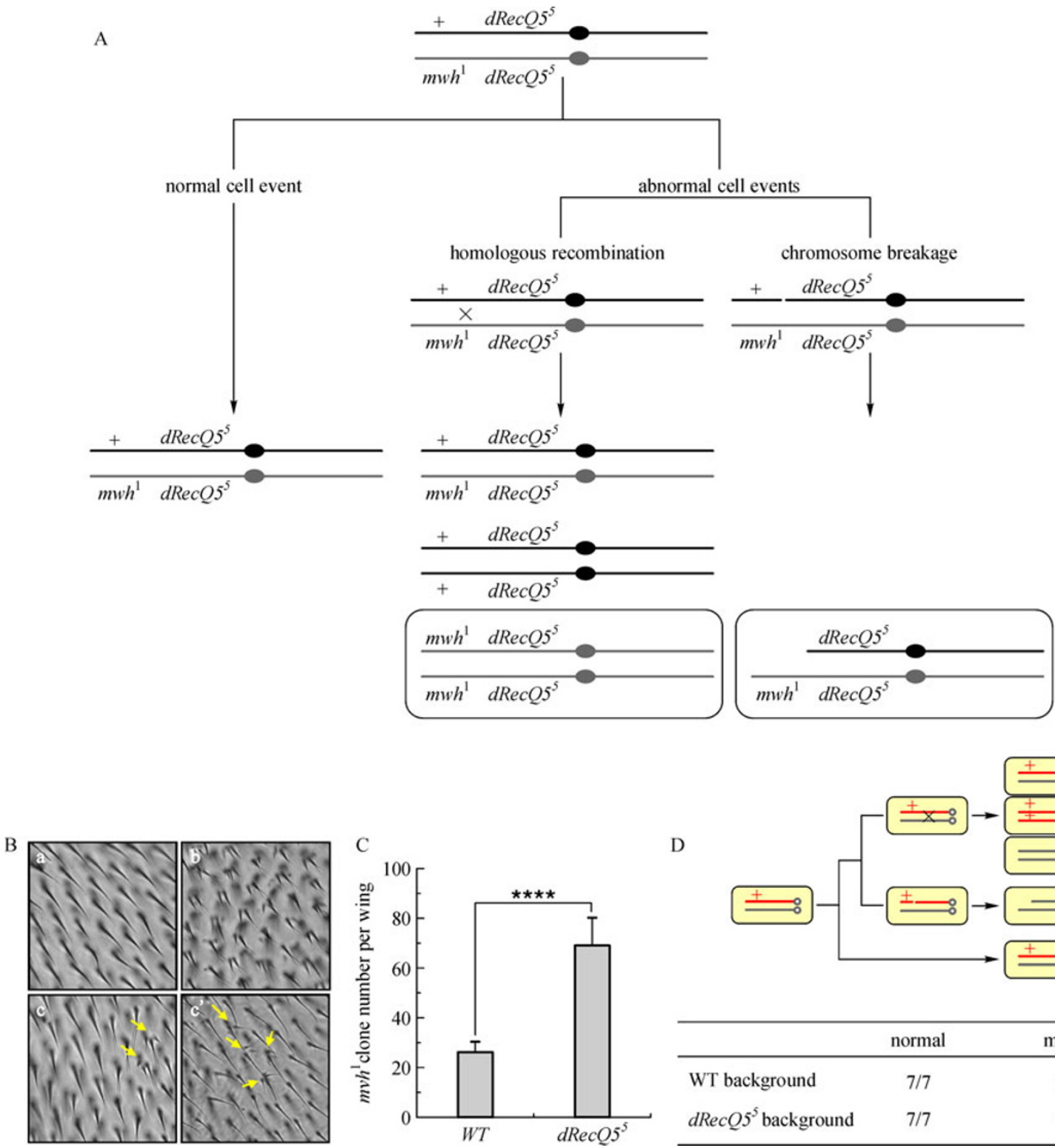

D

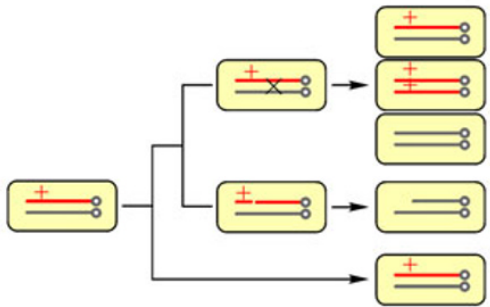

\begin{tabular}{lcc}
\hline & normal & mosaic \\
\hline WT background & $7 / 7$ & $0 / 7$ \\
dRecQ $5^{5}$ background & $7 / 7$ & $7 / 7$ \\
\hline
\end{tabular}

Figure 6. $d R e c Q 5$ mutants exhibit increased loss of heterozygosity (LOH). (A) The genetic basis of the LOH assay with $m w h^{1}$ as a marker. In the heterozygous $m w h$ animals, when the wild type copy of $m w h^{1}$ is lost during the proliferation of wing cell precursors, the resulting clone will exhibit the recessive mwh phenotype (multiple-wing hair). (B) dRecQ5 mutation leads to an increase in the number of $m w h^{1}$ mutant clones (arrows) resulting from homologous recombination. (a) Individual wing hairs in an animal heterozygous for $m w h^{1}$. Each hair follicle has one hair. (b) In an animal homozygous for $m w h^{1}$, each hair follicle has two or more hairs. (c and c') In an animal heterozygous for $m w h^{1}$ and homozygous for $d R e c Q 5$ mutation, individual cells that lost the wildtype $m w h$ gene due to homologous recombination show two or more hairs (arrows). (C) The frequency of $m w h^{1}$ clones per wing in $d R e c Q 5^{5}$ animals are significantly increased than in wild type. Nine wings for control and seven wings for $d R e c Q 5^{5}$ mutants were scored for $m w h^{1}$ clones. Data were analyzed by Student's t test and presented as mean \pm SEM with * for $p<0.05$, ${ }^{*}$ for $p<0.01$, and ${ }^{* * *}$ for $p<0.001$. (D) The genetic basis of the LOH assay with $w^{+}$as a marker (above). The numbers show the frequency of progenies with mosaic eyes. In wild type background, no flies with mosaic eyes were observed while in $d R e c Q 5^{5}$ background mosaic flies appeared in all tubes tested. 
dRecQ5 may participate in the process of SSA repair will require future investigations. One plausible possibility is that the strand annealing activity of RecQ5 (Ozsoy et al., 2001) may simply facilitate the annealing of the 3' ends of the direct repeats after resection of an induced DSB. However, dBLM is also reported to have such a strand annealing activity, and in the absence of $d B L M$ there exists a general shift of homologous recombination (HR-h, homology between homologous chromosomes) to SSA (Kappeler et al., 2008), which might suggest that strand annealing activity is not a key factor that determines the usage of SSA repair. Hu et al. (Hu et al., 2005) reported that RecQ5 and BLM have non-redundant roles in suppressing crossovers in mammalian cells. It remains to be determined how the induced DSBs may be repaired in the absence of both $d B L M$ and $d R e c Q 5$. Unfortunately, we were unable to construct a viable double mutant fly stock of $d B L M$ and $d R e c Q 5$ possibly due to a greater accumulation of un-repaired DNA damages.

Developmental defects caused by $d R e c Q 5$ mutation are evident in our study and also in a recent report by Nakayama et al. (Nakayama et al., 2009) in which the authors generated null alleles of $d R e c Q 5$ via $P$ element jump-out, recq $5^{D 1}$ and recq $5^{D 2}$, both of which show spontaneous mitotic defects and chromosomal aberrations. The chromosomal aberrations and cell cycle changes are likely to be the cause of developmental retardation (Fig. 2). This hypothesis is supported by the observation that induced DSBs by I-Sce I endonuclease cause severe lethality when un-repaired in both the F1-5 assay and w/w assay (Fig. 3C and Fig. 4D). Similar to $d R e c Q 5$, it has been reported that mutations in genes important for DNA damage repair and other related cellular processes can cause developmental defects (Brodsky et al., 2000; Xu et al., 2009).

In the F-R3 assay, the survival rate remains high both in wild type and $d R e c Q 5$ mutant animals, suggesting that the artificially induced DSBs by the cutting of I-Sce I enzyme can be efficiently repaired by either SDSA-mediated gap repair (Takeuchi et al., 2007) or non-homologous end joining regardless of the presence or absence of $d R e c Q 5$ function. However, in similar assays of F1-5, the survival rate (or DSB repair efficiency) is reduced by a loss of $d R e c Q 5$, with a more pronounced effect in males than in females (Fig. 4D). The F15 and F-R3 reporter constructs differ from each other in both the length of the homologous sequences that flank the I-Scel site (Takeuchi et al., 2007) and their chromosomal locations (F1-5 is located at 7E7 of the X chromosome while F-R3 is at $60 F 5$ of the second chromosome). These differences may contribute to the preference of pathways that will be used for the cells to repair the DSBs (Preston et al., 2006; Wei and Rong, 2007). This view is supported by the observation that in the $[w / w] 4 A$ assay, the animal survival rate also drops significantly in the presence of DSB induction (Fig. 3B), because the [w/w]4A construct, which is located on the second chromosome, unlike F-R3, offers homologous sequence for SSA repair in addition to NHEJ as shown in Fig. 5A. Furthermore, in F1-5 assay, the survival rate is different in males and females after DSB induction even in wild type background (Fig. 4D), may also suggest that successful DSB repair depends not only on the key repair machinery but also on the availability and type of homologous sequence and the location of the DSB at a given developmental stage (Preston et al., 2006; Wei and Rong, 2007).

In the current study, we used two assays, the mwh assay and the loss of $w^{+}$assay, to monitor the loss of $\mathrm{LOH}$ in Drosophila. Our results show that in the absence of $d R e c Q 5$ leads to a significant increase in LOHs (Fig. 6). First, this result is similar to what has been recently reported for $d W R$ Nexo (Saunders et al., 2008), suggesting that $d R e c Q 5$ and $d W R N$ exo are not functionally redundant in the process of homologous recombination. Secondly, deletion of RecQ5 does not lead to obvious increase of $\mathrm{LOH}$ in mammalian cells although it suppresses homologous recombination by interrupting Rad51 presynaptic filaments (Hu et al., 2007). Therefore, our finding may suggest that the functions of RecQ5 are not well conserved between flies and mammals. However, the precise mechanism of how $d R e c Q 5$ controls LOHs remains to be elucidated. We examined Rad51 responses to induced DSBs in the presence (wild type) and absence ( $d R e c Q 5$ mutants) of $d R e c Q 5$ function, no significant difference was detected as judged by Rad51 immunostainings (data not shown).

\section{MATERIALS AND METHODS}

\section{Fly stocks and genetics}

Flies were reared at $25^{\circ} \mathrm{C}$ on medium containing cornmeal, soybean, yeast, agar, syrup and molasses according to standard protocols. The fly stock of $S p / C y O P[U I E] 53 D$ was kindly provided by Dr. William R. Engels (Preston et al., 2006); the transgenic flies of F1-5 and F-R3 were kindly provided by Dr. Dieter Egli (Takeuchi et al., 2007); the fly stocks of [w/w]4A Sco/CyO, Sco/CyO;[w/w]2, [w/w]yellow Sb/TM3, Ser were generous gifts from Dr. Yikang. Rong (Wei and Rong, 2007). The rest of the fly stocks used in this study were from the Bloomington Drosophila Stock Center except for the $d R e c Q 5$ mutant allele (see below).

\section{Generation of the anti-dRecQ5 antibody}

The rabbit polyclonal antisera against dRecQ5 protein were raised using a recombinant polypeptide containing 106 amino acids of dRecQ5, ranging from a.a. 359 to a.a. 464 (YYGREDVRSIRFLLQNDAHRARGRGDKELLTERAIKQFEKITEFCERTTCRHKLFSDFFGDPTPDCSGQCDVCKRPKKAEKALEIFHRLCMDDAFKSHISLQDCAD). The corresponding cDNA fragment was first cloned into $\mathrm{pET} 28 \mathrm{~b}$ expression vector fused to a hexahistidine tag. Subsequently, the polypeptide expression and purification were carried out using the Ni-NTA resin (Qiagen) method following the manufacturer's instructions. The antibody was affinity-purified with protein A. 


\section{Generation and characterization of $d R e c Q 5$ mutants}

A $\sim 4.9 \mathrm{~kb} d R e c Q 5$ genomic fragment (coding region of the gene) and $a \sim 3 \mathrm{~kb} 5$ ' regulatory sequences with intended modifications of ATG site were cloned in the pTARG vector (Egli et al., 2006) to make the gene targeting donor construct, $p T A R G-d R e c Q 5$. Mutations of the ATG were introduced by PCR with the following oligos (changed bases are in italic) that were used for genomic DNA amplification. The primers used to amplify the $4.9 \mathrm{~kb} d R e c Q 5$ genomic fragment were 5'ataataagGCGGCCGCATGAAAGCGCTGTGCAC-3' and 5'tccCCGCGGATATGATCAAGATTGTGGAAC-3'. The primers for amplifying the upstream $3 \mathrm{~kb}$ fragment were 5'-cgACGCGTGGAACTCGAGAACTGACCACTC-3' and 5'-ataagaatGCGGCCGCACTTGGGCTGTTATTTAA-3'. Alteration of ATGGC to GCGGCC generates a Not I restriction site (Fig. 1). Oligos used to introduce the I-Sce I cleavage sequence that are inserted at the Afl II cutting site within the $4.9 \mathrm{~kb}$ fragment were 5'-TTAATAGGGATAACAGGGTAAT3'and 5'-TTAAATTACCCTGTTATCCCTA-3' (the underlined nucleotides indicate cleaved Afl II).

For generation of the $d R e c Q 5$ mutant, we used the ends-in gene targeting method (Rong et al., 2002; Egli et al., 2003). The flow chart of the targeting process is shown in Fig. 1A. Donor transgenic flies that bear the targeting construct on the second chromosome were crossed to flies that contain $h s-I-S c e ~ I$ and $h s-F L P$ transgenes. Three rounds of heat shock $\left(38^{\circ} \mathrm{C}, 1 \mathrm{~h}\right.$ each) were applied on days 2,3 and 4 after egg-laying. Heat-shocked virgins were singly crossed to $y$ w; eyFLP; MKRS/TM2, $y^{+}$males, and females were screened for targeted integration of targeting construct indicated by the $w^{+}$marker. Reduction of two dRecQ5 copies (one wild type and one mutant copy) by I-Cre I was performed by crossing the targeted alleles to $w^{1118}$; hs-l-Cre I, Sb/TM6. The offspring were given a single heat shock $\left(36^{\circ} \mathrm{C}, 1 \mathrm{~h}\right)$ at the third instar larval stage. $w^{-}$males were crossed individually to $y w$; Sp/CyO; MKRS/TM2, $y^{+}$to establish stocks. A specific pair of primers that was used to check mutations at the ATG site by Not I digestion of the PCR products is as follows: 5'CGCTTATAGGCGAGATGAATG-3' and 5'-TATACGATTCCGCAGCCTCT-3'. Not I digestion of the PCR products yields two fragments of $245 \mathrm{bp}$ and $846 \mathrm{bp}$ when the designed mutations appear, and one band of $1091 \mathrm{bp}$ for wild type flies. The allele, we designated $d \operatorname{Rec} Q 5^{5}$, was further confirmed by DNA sequencing for the designed mutations, and further analyzed throughout this study.

For dRecQ5 protein detection in both wild type and mutant flies, 0-4 $\mathrm{h}$ embryos of each genotype were collected and homogenized in RIPA buffer (150 mM NaCl, 50 mM Tris- $\mathrm{HCl}, 1 \%$ NP-40, 0.5\% Sodium deoxycholate, $1 \mathrm{mM}$ PMSF) in the presence of a protease inhibitor cocktail. $2 \times$ SDS buffer $(250 \mathrm{mM}$ Tris- $\mathrm{HCl}, \mathrm{pH} 6.8,40 \%$ glycerol, $2 \%$ SDS, $0.1 \%$ bromophenol blue, $20 \%$ 2-mercaptoethanol) was added to the extracts. Then the samples were boiled for $5 \mathrm{~min}$, and spun at max-speed at room temperature for $5 \mathrm{~min}$. The supernatants were applied for SDS-PAGE before transferred to PVDF membrane. The membranes were blocked for $1 \mathrm{~h}$ at room temperature and incubated with polyclonal dRecQ5 antibody $(1: 1000)$ overnight at $4^{\circ} \mathrm{C}$. Horseradish peroxidase-linked anti-rabbit IgG (1:3000) and Super Signal West Pico Trial Kit were used for signal detection according to standard manufacturer's instructions (Thermo Scientific, ECL-Kit).

\section{Physiological measurement assays}

The adult flies of $y w$ and $d R e c Q 5^{5}$ were used to collect embryos for $30 \mathrm{~min}$, and embryos were raised on agar plate containing agar/ sucrose/apple juice/ $\mathrm{H}_{2} \mathrm{O}(2.3 \mathrm{~g} / 2.5 \mathrm{~g} / 25 \mathrm{~mL} / 77 \mathrm{~mL})$. After $24 \mathrm{~h}$, the hatched first instar larvae were transferred to the standard food containing cornmeal, soybean, yeast, agar, syrup and molasses. Subsequently, 125 first instar larvae of each genotype were selected as a group to assay for the developmental stages.

\section{DSB sensitivity assays}

For gamma irradiation sensitivity tests, third instar larvae of both wild type and $d R e c Q 5$ mutants were treated with different doses of $\gamma$ irradiation $(0,10,20,25 \mathrm{~Gy})$ at a rate of $0.5 \mathrm{~Gy} / \mathrm{min}$ using ${ }^{60} \mathrm{Co}$ source. The irradiated larvae were transferred to a new food bottle. 100 irradiated larvae were placed in one bottle, and four parallel bottles were set out for each genotype. The number of eclosed flies of each bottle was scored and analyzed. For detection of the sensitivity of $d R e c Q 5^{5}$ to endogenously induced DSB damage by the endonuclease I-Sce I, 40 females of $y w$; Sp/CyO[UIE] in the presence or absence of $d R e c Q 5$ mutation were mated to $10 \mathrm{w}$; [w/w]4A Sco/CyO males with or without $d R e c Q 5$ mutation respectively, and the progenies of different crosses were scored and analyzed for endogenous DSB sensitivity. For the F1-5 and F-R3 assays, females of $y$ w F1-5; dRecQ5 (or $y$ w F1-5; +/+) and $y$ w; F-R3/CyO; dRecQ5 (or $y$ w; F-R3/CyO; +/+) were mated to $y$ w; Sp/CyO[UIE]; dRecQ5 (or y w; Sp/CyO[UIE]; +/+) males respectively. F1-5/+; +/CyO[UIE] and F$R 3 / C y O[U I E]$ flies represent the DSB-occurring fractions, and those flies without UIE were scored as the endogenous control independently. Survival rate was calculated as the ration of DSB-occurring fraction to the endogenous control fraction. For each experiment, six repeats were carried out. Data were analyzed by Student's $t$ test and presented as mean \pm SEM with * for $p<0.05$, ${ }^{* *}$ for $p<0.01$, and ${ }^{* * *}$ for $p<0.001$.

\section{Genome instability assay}

The mwh (multiple wing hairs) assay (Saunders et al., 2008) was used to examine the genome stability of $d R e c Q 5$ mutant flies. See Fig. $6 \mathrm{~A}$ for the principle of this experiment. For the detection of $m w h$ loss, fly wings with correct genotypes were dehydrated with isopropanol and mounted in media containing 1:1 methylsalicilate and Canada balsam (Sigma). All intervein wing hair cells were examined for the $m w h^{1}$ phenotype. Five to ten wings were examined for each genotype. $15 \mathrm{~Gy}$ of $\gamma$-irradiation was applied to make a genomic instability stress.

\section{In vivo DSB repair assays}

The w/w reporter system for assaying in vivo DSB repair has been described previously (Rong and Golic, 2003; Wei and Rong, 2007). The flies used in this study were [w/w]4A on chromosome 2 and [W/w]2 on chromosome 3 respectively. The line [w/w]yellow were derived from [w/w]2 by imperfect NHEJ (Wei and Rong, 2007) and used in combination with [W/W]2 in the homozygous assays (see Fig. 5E for schematic presentation). Another transgenic line $y$ w; $S p /$ CYO[UIE] was used to produce I-Sce I endonuclease to generated specific DSBs, [UIE] is the short-form for Ubiq::I-Sce I (Preston et al., 2006). Details of crossing procedure are as follows: for the hemizygous assay, $y w$; Sp/CyO[UIE] (;dRecQ5 $5^{5}$ ) virgins were crossed to $w$; [WIw] $4 A$ Sco/CyO (;dRecQ $\left.5^{5}\right)$ males, followed by selecting y w/Y; [WIw]4A Sco/CyO[UIE] (;dRecQ $5^{5}$ ) males to cross 
with $y$ w; Sp/CyO[UIE] (;dRecQ5 $5^{5}$ ) virgins. Sco, Sp progeny were scored for different repair products. For the homozygous assay, virgins of $y$ w; Sp/CyO[UIE] (; dRecQ5 $5^{5}$ ) were crossed to $w$; [w/w]yellow Sb/ $T M, \operatorname{Ser}\left(d R e c Q 5^{5}\right)$ males, followed by selecting y $w / Y$; +CyO[UIE]; [w/w]yellow $\mathrm{Sb} /+\left(d \operatorname{RecQ} 5^{5}\right)$ males to cross with $w$; Sco/CyO; [w/w]2 $\left(d R e c Q 5^{5}\right)$ virgins, from the offspring of which $w / Y$; Sco/CyO[UIE]; [w/w]yellow Sb/[w/w]2 $\left(d R e c Q 5^{5}\right)$ males were picked up to cross with y w virgins. Sco, $\mathrm{CyO}^{+}, \mathrm{Sb}^{+}$progeny were scored for different repair products.

\section{ACKNOWLEDGEMENTS}

We thank Drs William R. Engels, Yikang Rong, Dieter Egli, Walter Schaffner, Oleg Georgiev, Katsumi Kawasaki for fly stocks; Drs. Jim Kadonaga and Gary Karpen for antibodies; Drs Li Liu, Yikang Rong for stimulating discussions, Xuehong Liang and Wenbo Chen for their assistance. This work has been financially supported by the National Basic Research Program (973 Program) (Nos. 2009CB918702, 2005CB522804), the National Natural Science Foundation of China (Grant Nos. 30623005, 90608029 and 30771217) and Chinese Academy of Sciences (KSCX1-YW-R-70). We are grateful to the anonymous reviewers for their time and constructive suggestions.

\section{ABBREVIATIONS}

AED, after egg deposition; DSB, double strand break; GC, gene conversion; HEI, hybrid element insertion; HR, homologous repair; HR-h, homologous recombination between homologous chromosomes; DHJ, double holiday junction; LOH, loss of heterozygosity; NHEJ, non-homologous end joining; SDSA, synthesis dependent strand annealing; SSA, single strand annealing

\section{REFERENCES}

Adams, M.D., McVey, M., and Sekelsky, J.J. (2003). Drosophila BLM in double-strand break repair by synthesis-dependent strand annealing. Science 299, 265-267.

Bachrati, C.Z., and Hickson, I.D. (2008). RecQ helicases: guardian angels of the DNA replication fork. Chromosoma 117, 219-233.

Boubriak, I., Mason, P.A., Clancy, D.J., Dockray, J., Saunders, R.D., and Cox, L.S. (2009). DmWRNexo is a $3^{\prime}-5$ ' exonuclease: phenotypic and biochemical characterization of mutants of the Drosophila orthologue of human WRN exonuclease. Biogerontology 10, 267-277.

Brodsky, M.H., Sekelsky, J.J., Tsang, G., Hawley, R.S., and Rubin, G. M. (2000). mus304 encodes a novel DNA damage checkpoint protein required during Drosophila development. Genes Dev 14, 666-678.

Chu, W.K., and Hickson, I.D. (2009). RecQ helicases: multifunctional genome caretakers. Nat Rev Cancer 9, 644-654.

Egli, D., Selvaraj, A., Yepiskoposyan, H., Zhang, B., Hafen, E., Georgiev, O., and Schaffner, W. (2003). Knockout of 'metalresponsive transcription factor' MTF-1 in Drosophila by homologous recombination reveals its central role in heavy metal homeostasis. EMBO J 22, 100-108.

Egli, D., Yepiskoposyan, H., Selvaraj, A., Balamurugan, K., Rajaram, R., Simons, A., Multhaup, G., Mettler, S., Vardanyan, A., Georgiev, O., et al. (2006). A family knockout of all four Drosophila metallothioneins reveals a central role in copper homeostasis and detoxification. Mol Cell Biol 26, 2286-2296.

Ghabrial, A., Ray, R.P., and Schupbach, T. (1998). okra and spindle-B encode components of the RAD52 DNA repair pathway and affect meiosis and patterning in Drosophila oogenesis. Genes Dev 12, 2711-2723.

Hu, Y., Lu, X., Barnes, E., Yan, M., Lou, H., and Luo, G. (2005). Recql5 and BIm RecQ DNA helicases have nonredundant roles in suppressing crossovers. Mol Cell Biol 25, 3431-3442.

Hu, Y., Raynard, S., Sehorn, M.G., Lu, X., Bussen, W., Zheng, L., Stark, J.M., Barnes, E.L., Chi, P., Janscak, P., et al. (2007). RECQL5/Recql5 helicase regulates homologous recombination and suppresses tumor formation via disruption of Rad51 presynaptic filaments. Genes Dev 21, 3073-3084.

Jeong, S.M., Kawasaki, K., Juni, N., and Shibata, T. (2000). Identification of Drosophila melanogaster RECQE as a member of a new family of $\operatorname{RecQ}$ homologues that is preferentially expressed in early embryos. Mol Gen Genet 263, 183-193.

Jeong, Y.S., Kang, Y., Lim, K.H., Lee, M.H., Lee, J., and Koo, H.S. (2003). Deficiency of Caenorhabditis elegans RecQ5 homologue reduces life span and increases sensitivity to ionizing radiation. DNA Repair (Amst) 2, 1309-1319.

Johnson-Schlitz, D., and Engels, W.R. (2006). Template disruptions and failure of double Holliday junction dissolution during doublestrand break repair in Drosophila BLM mutants. Proc Natl Acad Sci U S A 103, 16840-16845.

Johnson-Schlitz, D.M., Flores, C., and Engels, W.R. (2007). Multiplepathway analysis of double-strand break repair mutations in Drosophila. PLoS Genet 3, e50.

Kappeler, M., Kranz, E., Woolcock, K., Georgiev, O., and Schaffner, W. (2008). Drosophila bloom helicase maintains genome integrity by inhibiting recombination between divergent DNA sequences. Nucleic Acids Res 36, 6907-6917.

Kawasaki, K., Maruyama, S., Nakayama, M., Matsumoto, K., and Shibata, T. (2002). Drosophila melanogaster RECQ5/QE DNA helicase: stimulation by GTP binding. Nucleic Acids Res 30, 3682-3691.

Kusano, K., Berres, M.E., and Engels, W.R. (1999). Evolution of the RECQ family of helicases: A drosophila homolog, Dmblm, is similar to the human bloom syndrome gene. Genetics 151, 1027-1039.

Kusano, K., Johnson-Schlitz, D.M., and Engels, W.R. (2001). Sterility of Drosophila with mutations in the Bloom syndrome genecomplementation by Ku70. Science 291, 2600-2602.

McVey, M., Andersen, S.L., Broze, Y., and Sekelsky, J. (2007). Multiple functions of Drosophila BLM helicase in maintenance of genome stability. Genetics 176, 1979-1992.

Nakayama, M., Yamaguchi, S., Sagisu, Y., Sakurai, H., Ito, F., and Kawasaki, K. (2009). Loss of RecQ5 leads to spontaneous mitotic defects and chromosomal aberrations in Drosophila melanogaster. DNA Repair (Amst) 8, 232-241.

Ozsoy, A.Z., Sekelsky, J.J., and Matson, S.W. (2001). Biochemical characterization of the small isoform of Drosophila melanogaster RECQ5 helicase. Nucleic Acids Res 29, 2986-2993.

Plessis, A., Perrin, A., Haber, J.E., and Dujon, B. (1992). Site-specific recombination determined by I-Scel, a mitochondrial group I intronencoded endonuclease expressed in the yeast nucleus. Genetics $130,451-460$. 
Preston, C.R., Flores, C.C., and Engels, W.R. (2006). Differential usage of alternative pathways of double-strand break repair in Drosophila. Genetics 172, 1055-1068.

Rong, Y.S., and Golic, K.G. (2003). The homologous chromosome is an effective template for the repair of mitotic DNA double-strand breaks in Drosophila. Genetics 165, 1831-1842.

Rong, Y.S., Titen, S.W., Xie, H.B., Golic, M.M., Bastiani, M., Bandyopadhyay, P., Olivera, B.M., Brodsky, M., Rubin, G.M., and Golic, K.G. (2002). Targeted mutagenesis by homologous recombination in D. melanogaster. Genes Dev 16, 1568-1581.

Rouet, P., Smih, F., and Jasin, M. (1994). Expression of a site-specific endonuclease stimulates homologous recombination in mammalian cells. Proc Natl Acad Sci U S A 91, 6064-6068.

Saunders, R.D., Boubriak, I., Clancy, D.J., and Cox, L.S. (2008). Identification and characterization of a Drosophila ortholog of WRN exonuclease that is required to maintain genome integrity. Aging Cell 7, 418-425.

Sekelsky, J.J., Brodsky, M.H., Rubin, G.M., and Hawley, R.S. (1999). Drosophila and human RecQ5 exist in different isoforms generated by alternative splicing. Nucleic Acids Res 27, 3762-3769.
Takeuchi, H., Georgiev, O., Fetchko, M., Kappeler, M., Schaffner, W., and Egli, D. (2007). In vivo construction of transgenes in Drosophila. Genetics 175, 2019-2028.

Trowbridge, K., McKim, K., Brill, S.J., and Sekelsky, J. (2007). Synthetic lethality of Drosophila in the absence of the MUS81 endonuclease and the DmBIm helicase is associated with elevated apoptosis. Genetics 176, 1993-2001.

Wei, D.S., and Rong, Y.S. (2007). A genetic screen for DNA doublestrand break repair mutations in Drosophila. Genetics 177, 63-77.

Wu, J., Capp, C., Feng, L., and Hsieh, T.S. (2008). Drosophila homologue of the Rothmund-Thomson syndrome gene: essential function in DNA replication during development. Dev Biol 323, 130-142.

Xu, Y., Lei, Z., Huang, H., Dui, W., Liang, X., Ma, J., and Jiao, R. (2009). dRecQ4 is required for DNA synthesis and essential for cell proliferation in Drosophila. PLoS One 4, e6107.

Zheng, L., Kanagaraj, R., Mihaljevic, B., Schwendener, S., Sartori, A. A., Gerrits, B., Shevelev, I., and Janscak, P. (2009). MRE11 complex links RECQ5 helicase to sites of DNA damage. Nucleic Acids Res 37, 2645-2657. 